\title{
Plastic embedded gold nanoparticles as 3D printing dichroic nanocomposite material
}

Lars Kool, Anton Bunschoten, Aldrik H. Velders, Vittorio Saggiomo*

Lars Kool, Dr. Anton Bunschoten, Prof. Aldrik H. Velders, Dr. Vittorio Saggiomo

Laboratory of BioNanoTechnology, Wageningen University, PO Box 8038, 6700, EK

Wageningen, The Netherlands

E-mail: vittorio.saggiomo@wur.nl

Keywords: 3D printing, nanocomposite, gold nanoparticles, dichroism

\begin{abstract}
:
Nanotechnology, even if unknowingly, has been used for millennia. The persistence of shiny colors in pottery and glass made hundreds and thousand of years ago is due to the use of nanoparticles in the fabrication of such ornaments. In the last decade, 3D printing has revolutionized fabrication and manufacturing processes, making it easier to produce, in a simple and fast way, 3D objects.

In this paper we show how to fabricate a $3 \mathrm{D}$ printable nanocomposite composed of dichroic gold nanoparticles and a 3D printable polymer. The minute amount of gold nanoparticles used for obtaining the dichroic effect does not influence the mechanical properties of the polymer nor its printability. Thus, the nanocomposite can be easily $3 \mathrm{D}$ printed using a standard $3 \mathrm{D}$ printer and shows a purple color in transmission and a brownish color in reflection.
\end{abstract}

\section{Introduction}

Since paleolithic cave painting, ${ }^{[1]}$ humans have always been fascinated by colors. Next to the traditional inorganic and organic colorants, nanoparticles received the lion's share, conferring shiny colors to pottery and glass in different eras. Copper nanoparticles, for example, have been found in red glass from the late Bronze Age, 1200-1000 BCE. ${ }^{[2]}$ The use of nanoparticles as a colorant boomed around the $4^{\text {th }}$ century CE within the Roman empire, where craftsmen, unaware of the existence of surface plasmon resonance, ${ }^{[3]}$ used metallic nanoparticles for coloring mosaic tiles, pottery and glass. ${ }^{[4,5]}$ 
Metallic nanoparticles were also used for staining glass during medieval times. Examples of which can still be found in many churches and cathedrals in Europe. The methodology for producing stained glass became reproducible in 1680 , when the method for producing the "ruby red glass" was born. ${ }^{[6]}$ The organic colorant of paintings from centuries ago is fading over time. However, potteries and glass embedded with nanoparticles made a thousand years ago are still the same shiny color as they were on the day of their production. Nanoparticles, in fact, are not subject to photobleaching, therefore, if the nanoparticles are stable over time, the color will persist for longer time with respect to organic colorants.

A single glass piece from the 4th century has puzzled scientists for long time: The Lycurgus cup. ${ }^{[7]}$ This cup has a very peculiar dichroic filter property, ${ }^{[8]}$ as its color is dependent on the illumination angle, changing from clear red (transmittance) to opaque green (reflectance). It was later found that this optical property was due to gold (AuNP) and silver nanoparticles (AgNP) of different sizes and shapes. ${ }^{[9,10]}$ However, only the Lycurgus cup, now stored in the British Museum, and six other broken pieces showing the same dichroic effect were found worldwide, hinting that achieving such optical effect was most probably due to serendipity rather than to master craftsmanship.

In recent years, 3D printing technology has revolutionized the prototyping and fabrication process, pushing the manufacturing of objects from factories to houses. Within the 3D printing world, scientists have also started modifying 3D printable plastics with, for example, catalysts ${ }^{[11]}$ or $\mathrm{TiO}_{2}$ nanoparticles ${ }^{[12]}$ to obtain new improved materials with special characteristics.

In this paper, we show how to fabricate a $3 \mathrm{D}$ printable dichroic material using gold nanoparticles, jumping from the $4^{\text {th }}$ century Roman glassmiths' methods to a modern and widely spread technology. 


\section{Results and discussion}

Dichroic AuNP were prepared using a modified Turkevich method, ${ }^{[13]}$ thus reducing gold ions to gold nanoparticles using citrate as both reducing and capping agent. In the classical Turkevich method, a boiling chloroauric acid solution is reacted with citrate using a molar ratio citrate to gold of 10, producing AuNP of around $10 \mathrm{~nm}$. When this ratio is changed, the size of the obtained nanoparticles changes as well. ${ }^{[14]}$ We discovered that a citrate/gold ratio between 0.6 and 0.8 produced dichroic nanoparticles that showed a brownish reflection and a purple transmission (Figure 1a). The nanoparticle solution was studied by transmission electron microscopy (TEM), showing that it was composed of polydisperse elongated nanoparticles of 50-60 $\mathrm{nm}$ with a mean aspect ratio of 1.4 (Figure 1b and S1). In addition to the surface plasmon resonance color, ${ }^{[15]}$ the large size of the nanoparticles increases the Mie scattering, ${ }^{[16]}$ giving rise to the opaque reflection. However, the elongated shape of the nanoparticles may also contribute to the dichroism, as nanoparticles with an aspect ratio bigger than 1.2 have been shown to possess dichroic properties. ${ }^{[17]}$ Even a bimodal size distribution of spherical nanoparticles may present dichroism as well.[18]
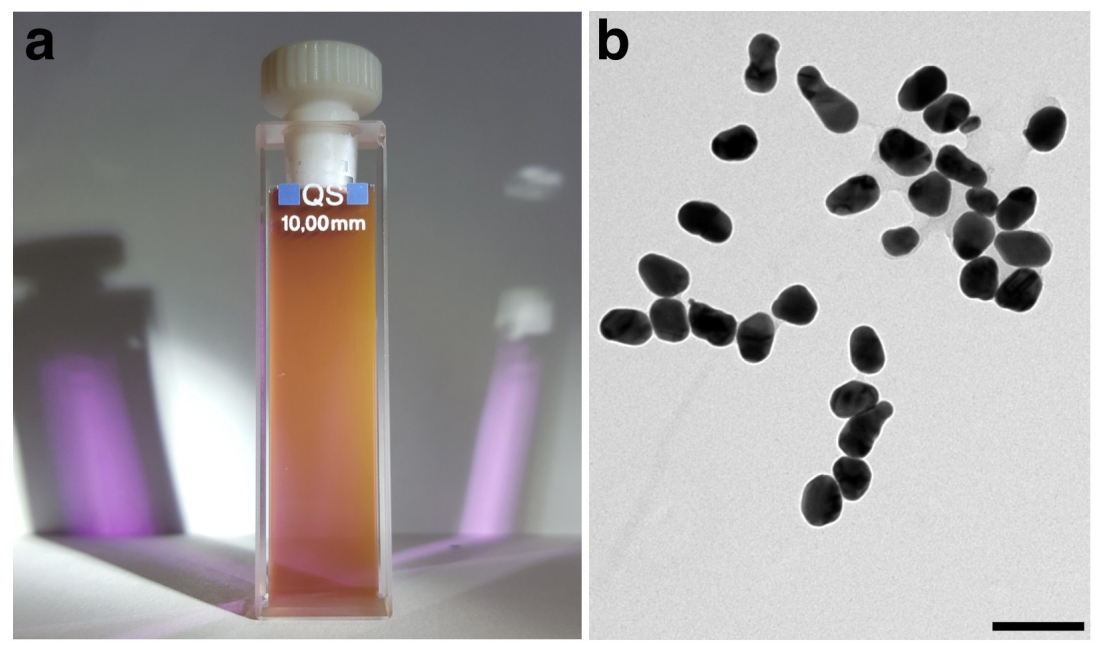

Figure 1. a) A dichroic AuNP solution, in the single picture it is possible to appreciate the brown reflection and purple transmission. b) TEM micrograph of the AuNP, scale bar 100nm. 
The presented synthesis is easy and fast, as it takes only few minutes to obtain the dichroic solution after the addition of the citrate. During the synthesis, the solution changed color multiple times: the yellow solution of the gold ions become blue one minute after the addition of the citrate solution. Two minutes later, the solution showed an intense black color, before becoming dichroic after another two minutes of boiling. The color changed during the synthesis hint that the dichroic nanoparticle formation is not just seeded growth, but a more complex mechanism. Therefore we studied the evolution of the nanoparticle formation over time using UV-Vis and TEM (S3-4). The time dependent study shows the formation of small gold nuclei that in time cluster together forming nanowire-like structures concomitant to the first color change. The second change of color, from ink-black to purple, is accompanied by an enhancement of the scattering, giving the purple solution a brown reflection. The explanation is that, while boiling, the gold nanowires fragment, creating nanoparticles with a large head and a slim and long tail, comparable to a tadpole. Over time the tail starts to shrink, due to intra-particle Ostwald ripening to minimize the total surface energy of the nanoparticle, yielding large oval dichroic gold nanoparticles. Both steps, namely chain growth and interparticle ripening have been previously reported in literature and are therefore not studied in depth here. ${ }^{[19,20]}$

The next step was to embed the dichroic AuNP in a 3D printable material, to be used with a standard off-the-shelf Fused Deposition Modeling (FDM) 3D printer. We chose to use polyvinyl alcohol (PVA) as the nanoparticle carrier, because it is one of the most used 3D printing materials, it is water soluble, thus mixable with the AuNP without need of changing solvent, and because it is known that PVA can be used as a capping agent for nanoparticles. ${ }^{[21]}$ 
3D printable grade PVA was then added to the dichroic solution for reaching a concentration of $0.1 \%(\mathrm{w} / \mathrm{w})$ of $\mathrm{AuHCl}_{4}(0.07 \%$ of gold in weight $)$ in PVA. The mixture was left at $70{ }^{\circ} \mathrm{C}$ in a ventilated oven until it was dry, yielding the AuNP-PVA nanocomposite. The so formed AuNP-PVA shows the same dichroic effect of the solution, brown opaque reflection and purple transparent transmission (Figure 2a, Video S1). Standard gold nanoparticles of less than $20 \mathrm{~nm}$ embedded in PVA do not show this dichroic property, as they give a red transparent color to the PVA, here named "ruby plastic" as reference to the first reproducible nanoparticle embedded glass "ruby glass" (Figure S8).

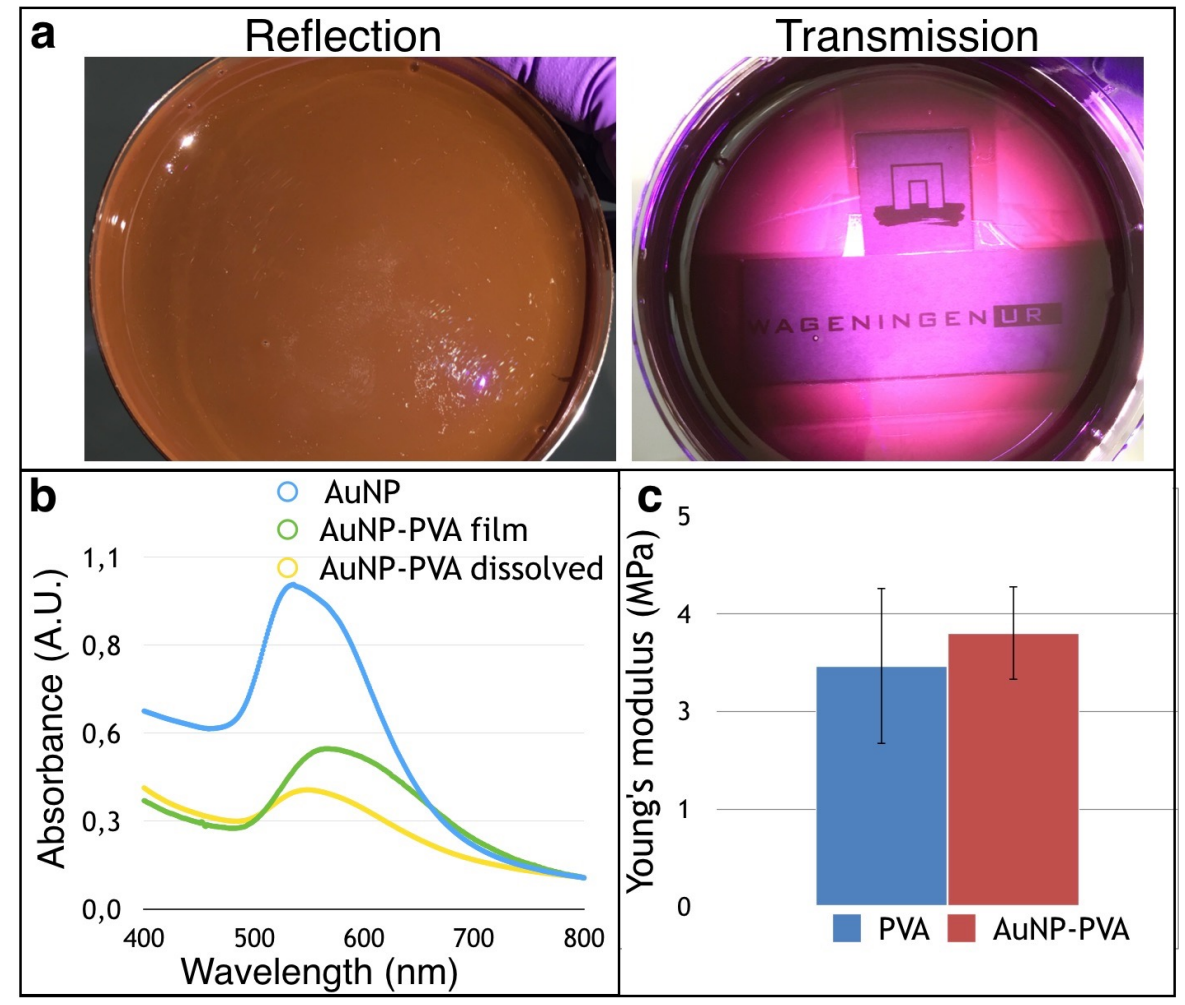

Figure 2. a) AuNP-PVA shows an opaque brown color in reflection and transparent purple in transmission. The transparency allows the readability of a text behind the plastic. b) UV-Vis spectra of the dichroic AuNP (blue), AuNP-PVA film (green) which shows a red shift probably due to a lack of solvent. When the AuNP-PVA film is dissolved in water, the AuNP shows again the same characteristic plasmon resonance band (yellow). c) Young's modulus of the AuNP-PVA compared to pure PVA.

The surface plasmon resonance band of the gold nanoparticles in the PVA film shows a redshift of $20 \mathrm{~nm}$ with respect to the original dichroic solution (Figure $2 \mathbf{b}$ ). As the 
concentration of gold atoms in the PVA is only $0.07 \%$, this shift cannot be attributed to the plasmon-plasmon coupling between nanoparticles, but it is probably due to the difference in the interaction between solvent and nanoparticles in water and when embedded in solid PVA. This effect is proven when the AuNP-PVA is dissolved in water releasing the nanoparticles, giving almost the same absorption band as the original dichroic solution (Figure $2 \mathrm{~b}$ and S6). The small difference in absorbance of $6 \mathrm{~nm}$ can be attributed to the capping ligand on the gold nanoparticles: citrate in the original dichroic solution and PVA in the AuNP-PVA dissolved in water.

We compared the TEM results of the original dichroic solution to the AuNP-PVA dissolved in water (Figure S5) to find that the nanoparticles were still of the same size and shape as the original ones, showing that the embedding in PVA does not influence the stability of the nanoparticles.

Lastly, we extruded the AuNP-PVA to fabricate a filament for FDM printing. As the percentage of $\mathrm{AuHCl}_{4}$ is only $0.1 \%$ with respect to PVA we did not envision a drastic change in the mechanical properties between pure PVA and AuNP-PVA. To test this, we 3D printed dog bone shaped strips of plastic $(2.5 \times 0.4 \times 0.1 \mathrm{~cm})$ and tested the elastic modulus (Young's modulus) using Dynamic Mechanical Analysis (DMA). The pure PVA and the AuNP-PVA, as expected, did not show significant differences in the elastic modulus giving an average of 3.5 +/- $0.8 \mathrm{MPa}$ and $3.8+/-0.6 \mathrm{MPa}$ respectively (Figure 2c). The relative high standard deviation error is given by the intrinsic layer by layer 3D fabrication method.

The filament was used for printing different " $21^{\text {st }}$ century Lycurgus cups" (Figure 3, S9-11 and Video S2). The small percentage of gold in the filament, also in this case, did not influence the printability of the plastic, and the same parameters for printing pure PVA were 
used successfully for printing AuNP-PVA. At the used percentage of gold in PVA, the dichroic effect in the printed objects can be detected when the wall thickness of the 3D printed object is bigger than $0.4 \mathrm{~mm}$. The 3D printed parts can also be smoothened by quickly washing the parts in water (Figure S12). The water solubility of PVA has, however, pros and cons: Due to its easily printable properties and water solubility, PVA is used mostly as supporting material. On the other hand, the water solubility of PVA is a problem when a strong resistant material is needed. For example one could not use the cup for drinking water as it will be dissolved after a few minutes. To overcome this problem, we coated the 3D printed cup in a layer of polydimethylsiloxane (PDMS), a flexible, non-toxic and food-safe transparent elastomer. ${ }^{[22]}$ The cup was brushed with liquid PDMS and it was cured at $70{ }^{\circ} \mathrm{C}$ for few hours. The PDMS coated AuNP-PVA cup was able to withstand water without any leakage (Figure S13 and Video S3). The cups, either coated or non-coated were stable at room temperature and ambient light for at least six months, without changing the dichroic effect.

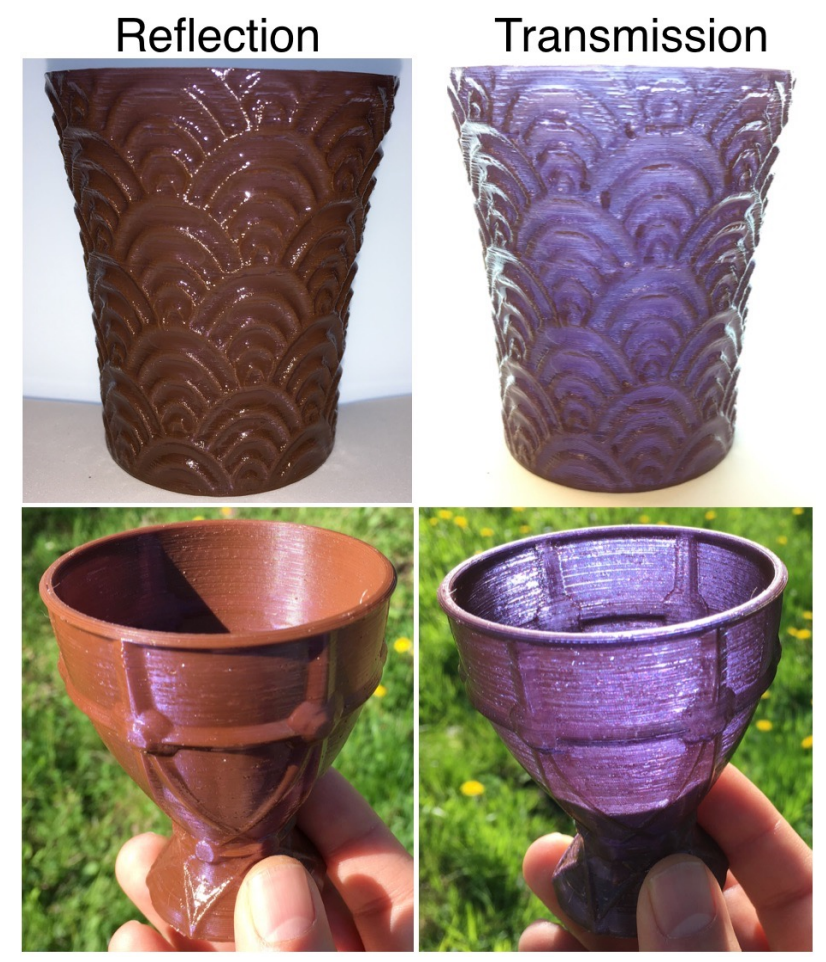

Figure 3. 3D printed cups using AuNP-PVA material showing the dichroic effect in artificial and sunlight. 


\section{Conclusions}

In conclusion, we showed how to synthesize and embed dichroic nanoparticles in 3D printable material. The AuNP-PVA nanocomposite is mechanically similar to the bare plastic and its dichroic optical properties are similar to the one shown by the AuNP solution. The 3D printed objects can be coated to achieve water impermeability and stability at room temperature for long time. We can envision this methodology to be used not only by artists, but also for studying optical properties of nanoparticles or, for example in 3D fabrication of optical filters.

\section{Material and methods}

\subsection{General:}

Chloroauric acid trihydrate and trisodium citrate dihydrate were obtained from Alfa Aesar and used without further purification. PVA $3 \mathrm{~mm}$ filament (Ultimaker) was obtained from Makerpoint. A Shimadzu UV1601 UV-Vis was used for the UV-Vis study. Samples were imaged in a JEOL 1400Plus TEM operating at $120 \mathrm{kV}$. The mean aspect ratio of the AuNP was calculated by measuring c.a. 800 particles from 24 micrographs. A TE instruments DMA Q800 was used for mechanical analyses of the plastics using the Stress/Strain program with a force ramp rate of $1 \mathrm{~N} / \mathrm{min}$ at isothermal $\left(25^{\circ} \mathrm{C}\right)$ temperature. Four samples for the pure PVA and four samples for the AuNP-PVA were tested, each sample was also flipped upside down and the stress/strain recorder again. The average of the measurements were used in Figure $2 \mathrm{c}$ with the standard deviation as error bar. 


\subsection{Synthesis of dichroic AuNP:}

$0.5 \mathrm{~mL}$ of a $34 \mathrm{mM}$ citrate solution in distilled water was added, in one shot, to a $100 \mathrm{~mL}$ boiling solution of $0.25 \mathrm{mM} \mathrm{HAuCl}_{4}$ in distilled water under vigorous stirring. The solution was left boiling while stirring for 5 minutes, until an brown reflection could be seen. The solution was cooled to room temperature and stored for further use. The solution can also be cooled to $50{ }^{\circ} \mathrm{C}$ to directly continue with the AuNP-PVA fabrication process.

\subsection{Fabrication of AuNP-PVA nanocomposite:}

Small pieces of PVA 3D printable filament were added to the dichroic solution at $50{ }^{\circ} \mathrm{C}$ to reach a final concentration of $0.1 \%(\mathrm{w} / \mathrm{w})$ of $\mathrm{AuHCl}_{4}$ in $\mathrm{PVA}$, taking in account that the maximum solubility of PVA in water is c.a. $20 \%(\mathrm{w} / \mathrm{w})$. A temperature below $50{ }^{\circ} \mathrm{C}$ produces less foam while gently stirring the PVA and it is, in general, easier to handle. When all the PVA is dissolved, the AuNP-PVA solution was transferred to plastic petri dishes and left in a ventilated oven until all the water was evaporated, yielding the hard AuNP-PVA plastic. Plastic petri dishes should be used instead of glass, as the PVA sticks to the glass as a glue, making the unmolding more difficult. The plastic was shredded and extruded to a $3 \mathrm{~mm}$ wire for the successive printing.

\subsection{D Printing:}

3D design of cups were downloaded from thingiverse.com: https://www.thingiverse.com/ thing:40770, https://www.thingiverse.com/thing:134879, https://www.thingiverse.com/thing: 1907150, and used under creative commons CC-BY-NC 3.0 and CC-BY-NC-SA 3.0.

The 3D designs were sliced with Cura 3.1.0 and printed on a Ultimaker 2+ FDM printer using a $0.4 \mathrm{~mm}$ nozzle $(0.2 \mathrm{~mm}$ layer height and $0.8 \mathrm{~mm}$ wall thickness $)$ and a $0.6 \mathrm{~mm}$ nozzle $(0.3$ 
$\mathrm{mm}$ height and $0.6 \mathrm{~mm}$ wall thickness). Printing parameters used were: Printing temperature $215^{\circ} \mathrm{C}$, build plate temperature $60{ }^{\circ} \mathrm{C}$, fan speed $50 \%$, print speed $50 \mathrm{~mm} / \mathrm{s}$.

Printed parts were smoothened by brushing the prints with a small wet brush. PDMS coated cups were made by mixing and stirring 10:1 Sylgard 184:curing agent. This solution was brushed on top of the cup and it was left standing at room temperature for $12 \mathrm{~h}$. Another coating of PDMS was done in the same way and then the cup was left in an oven at $70{ }^{\circ} \mathrm{C}$ for $2 \mathrm{~h}$.

\section{Supplementary Material}

Supplementary material is available from the publisher or the author.

\section{Acknowledgements}

We would like to thank Edward T.M. Berben (Philips Group Innovation, Research) for extruding the AuNP-PVA, Joshua A. Dijksman for supervising the mechanical measurements of the nanocomposite.

\section{References}

[1] E. Chalmin, M. Menu, C. Vignaud, Analysis of rock art painting and technology of Palaeolithic painters, Measurement Science and Technology, 14 (2003), 14.

[2] N. Brun, L. Mazerolles, M. Pernot, Microstructure of opaque red glass containing copper J. Mat. Sci. Lett. 10 (1991), 1418-1420.

[3] M. Faraday, The Bakerian Lecure, Experimental Relations of Gold to light, Philos. Trans. R Soc. Lond. 147 (1857), 145.

[4] F. J. Heiligtag, M. Niederberger, The fascinating world of nanoparticle research, Mat. Today, 16 (2013), 262-271. 
[5] "Nanoparticles in Ancient Materials: The Metallic Lustre Decorations of Medieval

Ceramics" P. Sciau, in The Delivery of Nanoparticles (Edited by A. A. Hashim), IntechOpen, United Kingdom, (2012), Ch. 25.

[6] I. Horn, D. von Kerssenbrock-Krosigk, G. Schulz, D. Syndram in Optical Technologies in the Humanities. Series of the International Society on Optics Within Life Sciences, (Edited by D. Dirksen, G. von Bally), vol 4. Springer, Berlin, Heidelberg, 1997. [7] I. Freestone, N. Meeks, M. Sax, C. Higgitt., The Lycurgus Cup - A Roman nanotechnology, Gold Bull., 40 (2007), 270-277.

[8] $P A C, \mathbf{2 0 0 7}, 79,293$ (Glossary of terms used in photochemistry, 3rd edition (IUPAC Recommendations 2006)) pg. 323.

[9] R. H. Brill, The chemistry of the Lycurgus Cup. in 7th International Congres of Glass. 1965. Brussels.

[10] D. Barber, I.C. Freestone, An investigation of the origin of the colour of the Lycurgus cup by analytical transmission electron microscopy, Archaeometry, 32 (1990), 33-45.

[11] J. S. Manzano, Z. B. Weinstein, A. D. Sadow, I. I. Slowing, Direct 3D Printing of Catalytically Active Structures, ACS Catal., 7 (2017), 7567-7577.

[12] M. R. Skorski, J. M. Esenther, Z. Ahmed, A. E. Miller, M. R. Hartings, Sci. Technol. Adv. Mat., The chemical, mechanical, and physical properties of 3D printed materials composed of TiO2-ABS nanocomposites, 17 (2016), 89-97.

[13] J. Turkevich, P. C. Stevenson, J. Hillier, Discussions of the Faraday Society, 1951, 11, 55.

[14] L. Shi, E. Buhler, F. Boué, F. Carn, How does the size of gold nanoparticles depend on citrate to gold ratio in Turkevich synthesis? Final answer to a debated question., J. Coll. Interf. Sci., 492 (2017), 191-198. 
[15] L. M. Liz-Marzán, Nanometals, formation and color, Mat. Today, 7 (2004), 26-31.

[16] X. Huang, M. A. El-Sayed, Gold nanoparticles: Optical properties and implementations in cancer diagnosis and photothermal therapy, J. Adv. Res., 1 (2010), 13-28.

[17] T. Som, B. Karmakar, Plasmonics, Surface Plasmon Resonance and Enhanced Fluorescence Application of Single-step Synthesized Elliptical Nano Gold-embedded Antimony Glass Dichroic Nanocomposites, 5 (2010), 149-159.

[18] R. H. Magruder III, S. J. Robinson, C. Smith, A. Meldrum, A. Halabica, R. F. Haglund, Jr., Dichroism in Ag nanoparticle composites with bimodal size distribution, J. App. Phy., 105 (2009), 024303.

[19] L. Pei, K. Mori, M. Adachi, Langmuir, Formation process of two-dimensional networked gold nanowires by citrate reduction of AuCl4- and the shape stabilization, 20, (2004), 7837-7843.

[20] X. Ji, X. Song, J. Li, Y. Bai, W. Yang, X. Peng, J. Am. Chem. Soc., Size Control of Gold Nanocrystals in Citrate Reduction: The Third Role of Citrate, 129 (2007), 13939-13948. [21] L. Longenberger, G. Mills, Formation of Metal Particles in Aqueous Solutions by Reactions of Metal Complexes with Polymers, J. Phys. Chem., 99 (1995), 475-478.

[22] A. Subramaniam, S. Sethuraman in Natural and Synthetic Biomedical Polymers (Ed S. G. Kumbar, C. T. Laurencin, M. Deng), Elsevier, 2014, Ch 18. 
The incorporation of dichroic nanoparticles in 3D printable plastic results in a dichroic nanocomposite material that can be easily 3D printed. Gold nanoparticles that shows

dichroic properties have been embedded in polyvinylalcohol, a standard 3D printable plastic. The nanocomposite shows dichroic properties and standard printability properties.

3D printing, nanocomposite, gold nanoparticles, dichroism

L. Kool, A. Bunschoten, A. H. Velders, V. Saggiomo*

Plastic embedded gold nanoparticles as 3D printing dichroic nanocomposite material

ToC figure:

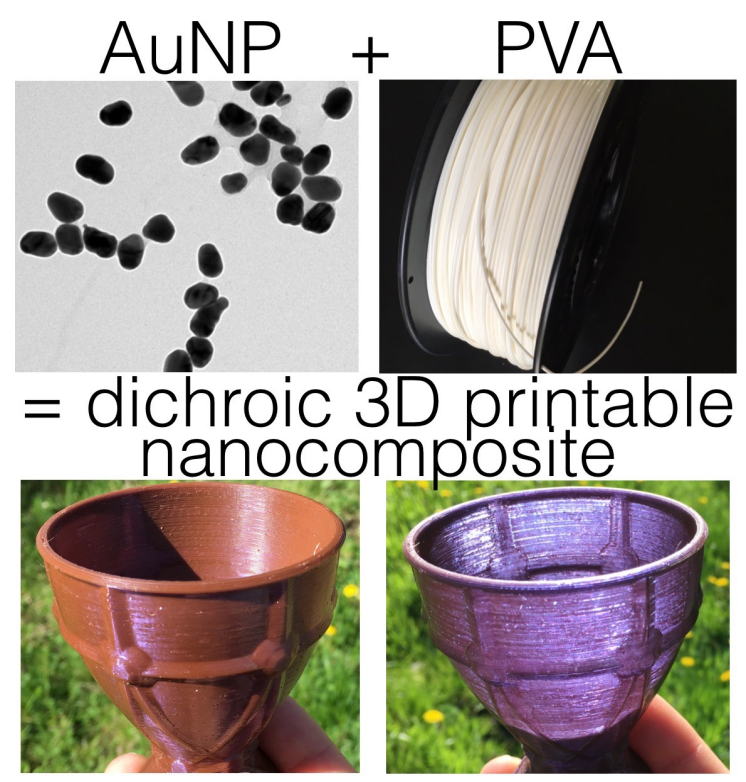


